TRANSACTIONS OF THE

AMERICAN MATHEMATICAL SOCIETY

Volume 184, October 1973

\title{
A METRIC CHARACTERIZATION OF CELLS
}

\author{
BY
}

\section{ELLARD NUNNALLY}

ABSTRACT. We examine finite dimensional compact convex metric spaces each having the property that the union of two line segments in the space, having more than one point in common, is a line segment. The question has been asked (Borsuk; Bing) whether each such space is a cell. The answer is yes if the dimension of the space is $\leq 2$ (Lelek and Nitka) or 3 (Rolfsen). Here we provide an affirmative answer for arbitrary finite dimension provided the space has the additional property that the join of any point to any line segment in the space is a convex set.

I. $\alpha$-metrics. A metric $d$ for a space $X$ is called convex if whenever $x$ and $y$ are points of $X$, there exists some point $p \in X$ such that $d(x, p)=1 / 2 d(x, y)=$ $d(y, p)$. Throughout this paper $X$ will denote a nondegenerate compact metric space with a convex metric $d$. If $p$ and $q$ are distinct points of $X$, then there is in $X$ an isometric image, $L(p, q)$, of the interval $[0, d(p, q)]$, having $p$ and $q$ as endpoints. The set $L(p, q)$ is called a line segment between $p$ and $q$.

Definition 1. The metric $d$ is called an $\alpha$-metric if whenever $L(p, q)$ and $L\left(p^{\prime}, q^{\prime}\right)$ are line segments in $X$ having more than one point in common, then $L(p, q) \cup L\left(p^{\prime}, q^{\prime}\right)$ is a line segment. (Note that if $L(p, q) \cup L\left(p^{\prime}, q^{\prime}\right)=L(s, t)$, then $\{s, t\} \subset\left\{p, q, p^{\prime}, q^{\prime}\right\}$.)

Throughout the remainder of this section, $d$ is assumed to be an a-metric.

There now follows several propositions and theorems concerning $\alpha$-metrics, mostly without proofs. The proofs are straightforward, and many are similar in spirit to those found in [3], [4], and [7].

Proposition 1. If $p$ and $q$ are distinct points of $X$, then $L(p, q)$ is unique.

Corollary. If $p$ and $q$ are distinct points of $X$, and $0 \leq r \leq 1$, then there is exactly one point $x \in X$ sucb that $d(p, x)=r d(p, q)$ and $d(q, x)=(1-r) d(p, q)$.

As in [4] we define $\lambda: X \times X \times[0,1] \rightarrow X$ as $\lambda(p, q, r)=$ the unique point $x \in$ $X$ such that $d(p, x)=r d(p, q)$ and $d(q, x)=(1-r) d(p, q)$. It is easy to check that $\lambda$ is continuous.

Received by the editors December 10, 1970 and, in revised form, April 14, 1972. AMS (MOS) subject classifications (1970). Primary 54F65. 
Definition 2. A line segment $L(p, m)$ is called a maximal ray from $p$ if whenever $L\left(p, m^{\prime}\right)$ is a segment such that $L(p, m) \subset L\left(p, m^{\prime}\right)$, then $L(p, m)=L\left(p, m^{\prime}\right)$, and hence $m=m^{\prime}$. A line segment $L(a, b)$ is called a maximal segment if whenever $L\left(a^{\prime}, b^{\prime}\right)$ is a segment such that $L(a, b) \subset L\left(a^{\prime}, b^{\prime}\right)$, then $L(a, b)=L\left(a^{\prime}, b^{\prime}\right)$ and hence $\{a, b\}=\left\{a^{\prime}, b^{\prime}\right\}$.

Theorem 1. Suppose $p$ and $q$ are distinct points of $X$. Then (1) there exists a unique maximal ray from $p, L(p, m)$, such that $L(p, q) \subset L(p, m)$; and (2) there exists a unique maximal segment $L(n, m)$ containing $L(p, q)$.

It follows from Proposition 1 and Theorem 1 that each pair of points of $X$ is contained in a unique maximal line segment. In fact, if $X$ is compact and $d$ is convex, then $d$ is an a-metric if and only if each pair of points of $X$ is contained in a unique maximal line segment.

Definition 3. If $A \subset X$ and $p \in X$, then the $p$-face of $A$, denoted by $F_{p}(A)$, is defined as $F_{p}(A)=\{a \in A \mid L(p, a) \cap A=\{a\}\}$. $\{p\}$.

Proposition 2. If $A \subset X, p \notin A,\{s, t\} \subset F_{p}(A), s \neq t$, then $L(p, s) \cap L(\phi, t)=$

Proof. Otherwise $L(p, s) \cup L(p, t)=L(p, s)$ (or $L(p, t)$ ). In which case $s \notin$ $F_{p}(A)$ (or $\left.t \notin F_{p}(A)\right)$.

Proposition 3. If $L$ is a maximal line segment and $p \notin L$, then $L=F_{p}(L)$.

If $A$ is a closed, convex subset of $X$, then $d$ is an $\alpha$-metric for $A$. That is, a closed convex subset of an " $\alpha$-space" is an " $\alpha$-space".

Definition 4. Suppose $A$ is a closed convex subset of $X$, By a rim point of $A$ we mean a point of $A$ which is an endpoint of some maximal segment in $A$. By the rim of $A$, denoted by $\partial(A)$, we mean the set of all rim points of $A$.

Theorem 2. Suppose $A$ is a closed subset of $X, p \in X-A$, and $A=F_{p}(A)$. Then $L(p, A)=\bigcup\{L(p, x) \mid x \in A\}$ is homeomorpbic to the cone over $A, C(A)$.

Proof. The obvious "linear" function from $C(A)$ to $L(p, A)$ is a homeomorphism.

In particular, if $A$ is an $n$-cell, $n \geq 0$, then $L(p, A)$ is an $(n+1)$-cell with $A \cup L(p$, bdry $A)=$ bdry $L(p, A)$.

Proposition 4. Suppose $A$ is a closed, convex subset of $X, L(s, t)$ is a maximal line segment in $X$, and $L(s, t) \cap A$ is nondegenerate. Then $L(s, t) \cap A$ is a maximal line segment in $A$.

Proposition 5. Suppose $A$ is a closed, convex subset of $X, p \in X-A, q \epsilon$ $A-F_{p}(A)$. Then $L(p, q)$ contains a point of $\partial(A)$ in its interior. 


\section{II. $\beta$-metrics.}

Definition 5. The metric $d$ on $X$ is called a $\beta$-metric if $d$ is an $\alpha$-metric having the property that if $L(a, b)$ is any line segment in $X$ and $p \in X$, then $L(p, L(a, b))$ is convex.

Throughout the remainder of this paper we assume that $d$ is a $\beta$-metric.

It might be well to indicate here the general thrust of the arguments to follow. Suppose, for the moment, that $X$ is finite dimensional. We will construct in $X$ a convex cell $l_{n}$ with a nonvoid interior. We then collapse $X$ into $I_{n}$ by means of a 1-1 function which takes $\partial(X)$ onto bdry $I_{n}$. In order to establish the continuity of $f$ we establish two important properties of $\partial(X)$. One is that any ray from a nonrim point of $X$ to a rim point contains no other rim point. The second is that $\partial(X)$ is closed. The remaining arguments are directed toward constructing the cell mentioned above and toward establishing these two properties of $\partial(X)$.

Proposition 6. If $A$ is a convex subset of $X$ and $p \in X-A$, then $L(p, A)$ is convex.

It follows from the continuity of $\lambda$ that if $A$ is closed, $L(p, A)$ is closed.

Theorem 3. Suppose $p$ and $q$ are distinct points of $X$, and $a \in X$ is not on the maximal line segment containing $p$ and $q$. (It follows from Propositions 3 and 6 and Theorem 2 that $L(a, L(p, q))$ is a convex 2-cell.) Then eacb maximal line segment in the 2-cell $A=L(a, L(p, q))$ bas its endpoints on the boundary of the cell.

Proof. Suppose $L\left(s, w^{\prime}\right)$ is a maximal segment in $A$ with $s \notin$ bdry $(A)$. Choose a point $w \neq s$ with $w \in L\left(s, w^{\prime}\right)$ and $w \notin$ bdry $(A)$. Then $L(w, s)$ is a maximal ray from $w$.

Define $F: \operatorname{bdry}(A) \times[0,1] \rightarrow A$ as follows: $F(x, t)=y$ where $y \in L(w, x)$ and $d(y, x) / d(w, x)=t$. Then $F$ is a contraction of the boundary of $A$ to $w$.

Now $F(x, t) \neq s$ for any $(x, t)$; for otherwise $s \in L(x, w)$, and hence, since $x \neq s, L(w, s)$ is a proper segment of $L(w, x)$, contradicting the maximal property of $L(w, s)$.

Hence we have a contraction of bdry $A$ to a point in $A-\{s\}$, which is impossible.

It follows from the above theorem that $\partial(A) \subset$ bdry $A$. Theorem 5 asserts $\partial(A)=$ bdry $A$.

Theorem 4. Suppose $p \in X-\partial(X)$ and $q \in \partial(X)$. Then $L(p, q) \cap \partial(X)=\{q\}$.

Proof. Suppose there exists an $s \in \partial(X) \cap L(p, q)$ and $s \neq q$. (See Figure 1.) Then $s \neq p$, for $p \notin \partial(X)$; and since $s \in \partial(X)$ there exists a point $t \in \partial(X)$ such that $L(s, t)$ is a maximal segment. 
(1) If $L$ is any segment which contains $p$ and $q$, then $t \notin L$. For if $t \in L$, then since $s \in L,\{s, t\} \subset L \cap L(s, t)$, and therefore $L \cup L(s, t)$ is a segment. And since $L(s, t)$ is maximal, $L \cup L(s, t)=L(s, t)$. Therefore $L$ is a subsegment of $L(s, t)$ containing $s$ as an interior point. Since this is impossible, $t \notin L$.

Let $L(a, b)$ denote the maximal segment containing $t$ and $p$; and let the order from $a$ to $b$ be $a \leq t<p<b$. (Again $t \neq p \neq b$, since $\{t, b\} \subset \partial(X)$.)

(2) Now $s \notin L(a, b)$. For if $s \in L(a, b)$, then $\{s, p\} \subset L(p, q) \cap L(a, b)$, and therefore $L(p, q) \cup L(a, b)$ is a segment. Since $L(a, b)$ is maximal, $L(p, q) \subset$ $L(a, b)$. Hence $L(a, b)$ is a segment containing $p, q$, and $t$, which contradicts (1). So $s \notin L(a, b)$.

By (2), $q \notin L(a, b)$, for otherwise $s \in L(a, b)$. So by Proposition $3, L(a, b)=$ $F_{q}(L(a, b))$; and hence, by Proposition $2, L(q, a) \cap L(q, p)=\{q\}$. Therefore we have

(3) $s \notin L(q, a)$.

Likewise,

(4) $s \notin L(q, b)$.

Now by Theorem $2, L(q, L(a, b))$ is a convex 2-cell with bdry $(L(q, L(a, b)))=$ $L(a, b) \cup L(q, a) \cup L(q, b)$. Also $L(s, t) \subset L(q, L(a, b))$, and by (2), (3), and (4), $s \notin$ bdry $(L(q, L(a, b)))$. Hence, by Theorem $3, L(s, t)$ is not maximal in $L(q, L(a, b))$, and therefore is not maximal in $X$. This contradiction proves the theorem.

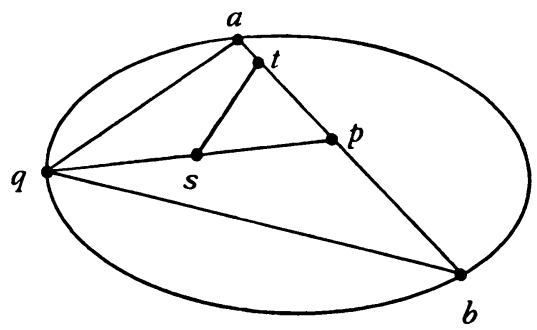

Figure 1

Theorem 5. Suppose $A$ is a nondegenerate closed convex subset of $X$, $A-\partial(A) \neq \varnothing, p \in X-A$, and $A=F_{p}(A)$. Then $\partial(L(p, A))=L(p, \partial(A)) \cup A$.

Before proving Theorem 5, if $p \neq a \in L(p, A)$ define the projection of $a$ on $A$, denoted by $p(a)$, to be that unique point $a^{\prime} \in A$ such that $a \in L\left(p, a^{\prime}\right)$. It is easily shown that the map $p$ is "linear" in the sense that if $p \notin L(a, b) \subset L(p, A)$, then $p(L(a, b))=L(p(a), p(b))$.

Proof of Theorem 5. First we show $\partial(L(p, A)) \subset L(p, \partial A) \cup A$ by showing that if $L(a, b)$ is a maximal segment in $L(p, A)$, then $\{a, b\} \subset L(p, \partial A) \cup A$.

We may assume $p \notin\{a, b\}$, for otherwise $\{a, b\} \subset\{p\} \cup A \subset L(p, \partial A) \cup A$. Let 
$p(a)=a^{\prime} \neq b^{\prime}=p(b)$ and let $L(x, y)$ be the maximal segment in $A$ containing $\left\{a^{\prime}, b^{\prime}\right\}$. Since $L(a, b)$ is a maximal segment in the convex 2-cell $L(p, L(x, y))$, by Theorem 3, $\{a, b\} \subset L(x, y) \cup L(p, x) \cup L(p, y) \subset L(p, \partial A) \cup A$.

Now we show $L(p, \partial A) \cup A \subset \partial(L(p, A))$. Suppose $a \in L(p, \partial A) \cup A$. Since it easily follows that $L(p, x)$ is a maximal segment in $L(p, A)$ for each $x \in A$, we assume $a \notin A, a \neq p$. Let $p(a)=a^{\prime} \epsilon \partial A$ and select $x \in A-\partial A$. It suffices to show that $L(x, a)$ is a maximal ray from $x$ in $L(p, A)$.

Suppose, on the contrary, that $L(x, s)$ is a maximal ray from $x$ in $L(p, A)$ containing $L(x, a)$ with $a \neq s$. Then $s \in \partial(L(p, A)) ; s \neq p$ (otherwise $a^{\prime}=x \in \partial A$ ); and $s \notin A$. Hence $p(s)=s^{\prime} \in \partial A$. Also $s^{\prime} \neq a^{\prime}$, for otherwise $\{s, a\} \subset L\left(p, a^{\prime}\right) \cap$ $L(x, s)$ and therefore $L\left(p, a^{\prime}\right) \cup L(x, s)=L\left(p, a^{\prime}\right)$. Then $x=a^{\prime}$.

Now since $p(L(x, s))=L\left(x, s^{\prime}\right)$, we have $a^{\prime} \in L\left(x, s^{\prime}\right)$. This violates Theorem 4. Hence $L(x, a)$ is a maximal ray from $x$ in $L(p, A)$.

Corollary. Suppose $\left\{p_{i}\right\}_{i=0}^{n}$ are distinct points of $X$. Set $A_{0}=\left\{P_{0}\right\}$ and for $1 \leq i \leq n$ set $A_{i}=L\left(p_{i}, A_{i-1}\right)$. Suppose also $F_{p_{i}}\left(A_{i-1}\right)=A_{i-1}$ for $1 \leq i \leq n$ so that, by Theorem $2, A_{i}$ is an i-cell. Then $\partial\left(A_{i}\right)=$ bdry $A_{i}$.

Proof. Induction on $i$, together with the remark following Theorem 2.

Theorem 6. Suppose $A$ is a closed convex subset of $X, p \in X-A$ and the sets $F_{p}(A)$ and $A-\partial A$ bave a point $q$ in common. Then $F_{p}(A)=A$.

Proof. Suppose $x \in A, x \neq q$. We show $x \in F_{p}(A)$. Let $L(s, t)$ be the maximal segment in $X$ containing $\{x, q\}$. By Proposition $4, L(s, t) \cap A=L(a, b)$ is a maximal segment in $A$. To be specific we take the order on $L(a, b)$ as $a<q<x \leq b$ (see Figure 2).

Since $q \in F_{p}(A), p \notin L(s, t)$. Hence $S=L(p, L(a, x))$ is a convex 2-cell with bdry $S=L(p, a) \cup L(a, x) \cup L(p, x)$. Now if $x \notin F_{p}(A)$, there exists a point $w \epsilon$ $A \cap L(p, x)$ with $w \neq x$. Hence the arc $L(a, w)$ which lies, except for its endpoints, in the interior of $S$ and separates $p$ and $q$ in $S$ also lies in $A$. Therefore $L(p, q)$ contains a point of $A$ other than $q$, which contradicts the fact that $q \epsilon$ $\boldsymbol{F}_{p}(A)$.

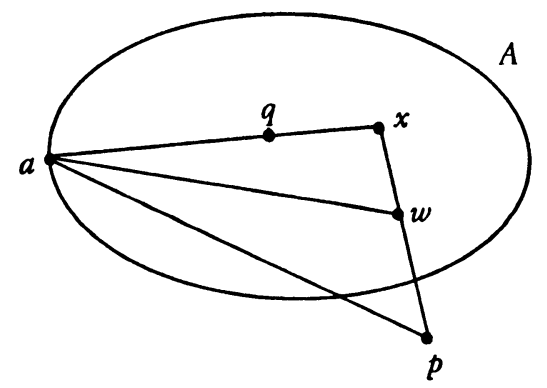

Figure 2 
Proposition 7. Suppose $A$ is a closed convex subset of $X,\left\{q_{1}, q_{2}\right\} \subset A, q_{1} \neq$ $q_{2}, p \in X-A$ and is not on any line segment containing $\left\{q_{1}, q_{2}\right\}$, and $q_{1} \notin F_{p}(A)$. If $a \in L\left(q_{1}, q_{2}\right), a \notin\left\{q_{1}, q_{2}\right\}$, then $a \notin F_{p}(A)$.

Proof. Very much like that for Theorem 6.

Theorem 7. If $A$ is a closed, convex subset of $X,\left\{p_{1}, p_{2}\right\} \subset X, p_{1} \neq p_{2}$, with $L\left(p_{1}, p_{2}\right) \cap A=a \in A-\partial(A)$, then $B=L\left(p_{1}, A\right) \cup L\left(p_{2}, A\right)$ is closed, convex and $\partial B=L\left(p_{1}, \partial A\right) \cup L\left(p_{2}, \partial A\right)$.

Proof. First if $p_{1} \in A$, then $p_{1}=a \in F_{p_{2}}(A)$. Hence, by Theorem $6, A=F_{p_{2}}(A)$. Then by Theorem $5, B=A \cup L\left(p_{2}, A\right)=L\left(p_{2}, A\right)$ is closed, convex and $\partial(B)=$ $A \cup L\left(p_{2}, \partial(A)\right)=L\left(p_{1}, \partial(A)\right) \cup L\left(p_{2}, \partial(A)\right)$. So we now suppose $\left\{p_{1}, p_{2}\right\} \subset X-A$, and hence $F_{p_{1}}(A)=A=F_{p_{2}}(A)$.

Lemma 1. $F_{p_{2}}\left(L\left(p_{1}, A\right)\right)=A$.

Proof. In view of Theorem 6 it is sufficient to show that if $q \in L\left(p_{1}, A\right)-A$, then $q \notin F_{p_{2}}\left(L\left(p_{1}, A\right)\right)$.

It follows from Theorem 6 and the fact that $p_{1} \notin F_{p_{2}}\left(L\left(p_{1}, A\right)\right)$ that if $q \epsilon$ $L\left(p_{1}, A\right)-\partial L\left(p_{1}, A\right)$, then $q \notin F_{p_{2}}\left(L\left(p_{1}, A\right)\right)$.

So suppose $p_{1} \neq q \in \partial L\left(p_{1}, A\right)-A=\left(A \cup L\left(p_{1}, \partial(A)\right)-A=L\left(p_{1} \partial A\right)-\partial A\right.$. We may select a point $q_{1} \in \partial A, q \neq q_{1}$, with $q \in L\left(p_{1}, q_{1}\right)$. Since $q_{1} \neq a, p_{2}$ is not on any line segment containing $p_{1}$ and $q_{1}$. Hence by Proposition $7, q \notin F_{p_{2}}\left(L\left(p_{1}, A\right)\right)$. This proves Lemma 1.

From Lemma 1 it follows that $L\left(p_{2}, L\left(p_{1}, A\right)\right)=L\left(p_{2}, F_{p_{2}}\left(L\left(p_{1}, A\right)\right)\right) \cup$ $L\left(p_{1}, A\right)=L\left(p_{2}, A\right) \cup L\left(p_{1}, A\right)=B$ is closed and convex.

Lemma 2. Each line segment in B which contains two points of A must lie in $A$.

Proof. This follows easily from the fact that $F_{p_{i}}(A)=A$.

Lemma 3. We now show that if $x \in A-\partial(A)$ then $L\left(p_{i}, x\right)$ is not maximal in $B, i=1$, 2. (In particular $L\left(p_{i}, x\right) \subset L\left(p_{i}, x_{0}\right)$ with $x \neq x_{0}, x_{0} \in B$.)

Proof. If $x=a, L\left(p_{i}, x\right)$ is properly contained in $L\left(p_{1}, p_{2}\right)$; so suppose $x \neq a$.

Let $L(s, t)$ be the maximal segment in $A$ containing $a$ and $x$. Since $\{s, t\} \cap$ $\{a, x\}=\varnothing$, let the order on $L(s, t)$ be $s<a<x<t$, and let $S=L\left(t, L\left(\phi_{1}, p_{2}\right)\right)$. This is a convex 2-cell with $\partial S=$ bdry $S=L\left(p_{1}, p_{2}\right) \cup L\left(t, p_{1}\right) \cup L\left(t, p_{2}\right)$.

Since $\{a, t\} \subset S, x \in S$. Hence $L\left(p_{i}, x\right) \subset S$. If $L\left(p_{i}, x\right)$ were maximal in $B$, $L\left(p_{i}, x\right)$ would be maximal in $S$. But since $x \notin \partial S$ we must have $L\left(p_{i}, x\right) \subset$ $L\left(p_{i}, x_{0}\right)$ for some $x_{0} \neq x, x_{0} \in S \subset B$. This proves Lemma 3. 
Lemma 4. $A$ separates $B$.

Proof. This follows easily from the observation that $L\left(p_{1}, A\right) \cap L\left(p_{2}, A\right)=A$. We are now ready to show $\partial B \subset L\left(p_{1}, \partial A\right) \cup L\left(p_{2}, \partial A\right)$. Suppose $L(s, t)$ is maximal in $B$; we show $\{s, t\} \subset L\left(p_{1}, \partial A\right) \cup L\left(\phi_{2}, \partial A\right)$.

In view of Lemmas 2,4 and Theorem 5 , we may assume that $L(s, t)$ contains exactly one point $q$ of $A$.

Case I. $q \notin\{s, t\}$. If $\{s, t\} \subset L\left(p_{1}, A\right)$, then $L(s, t)$ is maximal in $L\left(p_{1}, A\right)$. Hence $\{s, t\} \subset \partial L\left(p_{1}, A\right)-A=\left(L\left(p_{1}, \partial A\right) \cup A\right)-A \subset L\left(p_{1}, \partial A\right)$. So suppose $s \epsilon$ $L\left(p_{1}, A\right)$ and $t \in L\left(p_{2}, A\right)$. Then $L(s, q)=L(s, t) \cap L\left(p_{1}, A\right)$ is maximal in $L\left(\phi_{1}, A\right)$, by Proposition 4 , and $L(q, t)=L(s, t) \cap L\left(p_{2}, A\right)$ is maximal in $L\left(p_{2}, A\right)$. Hence $s \in \partial L\left(p_{1}, A\right)-A \subset L\left(p_{1}, \partial A\right)$ and $t \in \partial L\left(\phi_{2}, A\right)-A \subset L\left(\phi_{2}, \partial A\right)$.

Case II. Suppose $q=t$, and $s \in L\left(p_{1}, A\right)-A$. Then $L(s, t)$ is maximal in $L\left(p_{1}, A\right)$ and hence $s \in L\left(\phi_{1}, \partial A\right)$. We now show $q \in \partial A \subset L\left(p_{1}, \partial A\right)$. Suppose $q \notin$ $\partial A$. Let $s^{\prime} \in \partial A$ such that $s \in L\left(p_{1}, s^{\prime}\right)$. We have $s^{\prime} \neq q$. Let $L(x, y)$ be the maximal segment in $A$ containing $\left\{s^{\prime}, q\right\}$, and let the order be $x \leq s^{\prime}<q<y$. Choose $r$ so that $q<r<y$. Since $q \notin \partial A$ and $y \in \partial A$ by Theorem $4, r \notin \partial A$. Hence, by Lemma 3, there exists an $r_{0} \in B$ such that $L\left(p_{1}, r\right) \subset L\left(p_{1}, r_{0}\right)$ and $r \neq r_{0}$.

Now consider $S=L\left(x, L\left(p_{1}, r_{0}\right)\right)$. Since $\{x, r\} \subset S, q \in S$; and since $L\left(p_{1}, s^{\prime}\right) \subset S, s \in S$. Hence $L(s, q) \subset S$, and hence is maximal in $S$. So $q \in \partial S$. Now $\partial S=$ bdry $S=L\left(p_{1}, r_{0}\right) \cup L\left(x, p_{1}\right) \cup L\left(x, r_{0}\right)$. Since $r \in L\left(p_{1}, r_{0}\right), q \notin$ $L\left(p_{1}, r_{0}\right)$ (otherwise not both $r$ and $q$ are in $F_{p_{1}}(A)=A$ ). Likewise $q \notin L\left(x, p_{1}\right)$.

Since $r \neq r_{0}$, and since $x$ is not on any line segment containing $p_{1}$ and $r_{0}$ (otherwise not both $x$ and $r$ could be in $F_{p_{1}}(A)$ ), $L(x, r) \cap L\left(x, r_{0}\right)=\{x\}$. So since $q \in L(x, r), q \notin L\left(x, r_{0}\right)$. Hence $q \notin \partial S$. This contradiction establishes Case II. Therefore $\partial B \subset L\left(\phi_{1}, \partial A\right) \cup L\left(p_{2}, \partial A\right)$.

To see that $L\left(p_{1}, \partial A\right) \cup L\left(p_{2}, \partial A\right) \subset \partial B$, suppose $x \in L\left(p_{1}, \partial A\right)$. We show $x \epsilon$ $\partial B$. Since $L\left(p_{1}, p_{2}\right)$ is maximal in $B$, suppose $x \neq p_{1}$.

If $x \in \partial A$, it follows from Lemma 2 that $x \in \partial B$. If $x \notin \partial A$, then as, in the proof of Theorem $5, L(a, x)$ is a maximal ray from $a$ in $L\left(p_{1}, A\right)$. Hence, by Lemmas 2 and $4, L(a, x)$ is a maximal ray from $a$ in $B$. Therefore $x \in \partial B$.

Theorem 8. If $X$ is finite dimensional, then $\partial(X)$ is closed.

Proof. Suppose $x_{0} \in X-\partial(X)$. Let $l_{1}=L\left(p_{0}, p_{1}\right)$ be any maximal line segment containing $x_{0}$. Since $x_{0} \notin \partial(X), p_{0} \neq x_{0} \neq p_{1}$.

Either $l_{1}$ contains a neighborhood of $x_{0}$, or it does not. Suppose it does not. Choose $\epsilon>0$ so that $S_{\epsilon}\left(x_{0}\right)$ misses $\left\{p_{0}, p_{1}\right\}$. Select a point $x \in S_{\epsilon}\left(x_{0}\right)$ with $x \notin$ $I_{1}$. Since $L\left(x, x_{0}\right) \subset S_{\epsilon}\left(x_{0}\right), L\left(x, x_{0}\right) \cap \partial I_{1}=L\left(x, x_{0}\right) \cap\left\{p_{0}, p_{1}\right\}=\varnothing$. Hence by Proposition 5, $x_{0} \in F_{x}\left(I_{1}\right)$ and therefore $L\left(x, x_{0}\right) \cap I_{1}=\left\{x_{0}\right\}$. 
Let $L\left(p_{2}, p_{3}\right)$ be the maximal segment in $X$ containing $\left\{x, x_{0}\right\}$. Then $L\left(p_{2}, p_{3}\right) \cap I_{1}=\left\{x_{0}\right\}$ and $p_{2} \neq x_{0} \neq p_{3}$. Set $I_{2}=L\left(p_{2}, I_{1}\right) \cup L\left(p_{3}, I_{1}\right)$. By Theorem $7, I_{2}$ is closed, convex and $\partial I_{2}=L\left(p_{2}, \partial I_{1}\right) \cup L\left(p_{3}, \partial I_{1}\right)=L\left(p_{2},\left\{p_{0}, p_{1}\right\}\right) \cup$ $L\left(p_{3},\left\{p_{0}, p_{1}\right\}\right)$. Also $I_{2}$ is 2-dimensional, since it is the sum of two 2-cells.

If $I_{2}$ contains no neighborhood of $x_{0}$, then choose $\epsilon>0$ so that $S_{\epsilon}\left(x_{0}\right)$ misses $\partial I_{2}$ and select a point $y \in S_{\epsilon}\left(x_{0}\right)-I_{2}$. Again since $L\left(y, x_{0}\right) \subset S_{\epsilon}\left(x_{0}\right), L\left(y, x_{0}\right)$ misses $\partial I_{2}$ and thus $x_{0} \in F_{y}\left(I_{2}\right)$; hence by Theorem $6, I_{2}=F_{y}\left(I_{2}\right)$. Let $L\left(p_{4}, p_{5}\right)$ be the maximal segment in $X$ containing $\left\{y, x_{0}\right\}$. Set $I_{3}=L\left(p_{4}, I_{2}\right) \cup L\left(\phi_{5}, I_{2}\right)$. Then $I_{3}$ is closed, convex and $\partial I_{3}=L\left(\phi_{4}, \partial I_{2}\right) \cup L\left(p_{5}, \partial I_{2}\right)$ and since $p_{4} \neq x_{0} \neq$ $p_{5}, x_{0} \notin \partial I_{3}$. Also $I_{3}$ is 3-dimensional, since it is the sum of four 3-cells.

If $l_{3}$ contains no neighborhood of $x_{0}$, we repeat the process until eventually, since $X$ is finite dimensional, we arrive at a closed convex set $I_{n}$ with $x_{0} \epsilon$ $I_{n}-\partial I_{n}$ and $\epsilon>0$ such that $S_{\epsilon}\left(x_{0}\right) \subset I_{n}-\partial I_{n} \subset X-\partial X$.

Theorem 9. If $X$ is finite dimensional, then $X$ is bomeomorphic to an n-cell for some $n \geq 1$.

Proof. The proof is similar in many respect to that of Theorem 8.

Let $p_{0} \neq p_{1}$ be any two points of $X$. Let $a_{1}$ denote the midpoint of $L\left(p_{0}, p_{1}\right)=I_{1}$. Either $I_{1}$ contains a neighborhood of $a_{1}$ or it does not.

If it does not, choose $\epsilon>0$ so that $S_{\epsilon}\left(a_{1}\right) \cap \partial I_{1}=S_{\epsilon}\left(a_{1}\right) \cap\left\{p_{0}, p_{1}\right\}=\varnothing$. Then select a point $p_{3} \in S_{\epsilon}\left(a_{1}\right)-I_{1}$. As in the proof of Theorem $8, L\left(p_{3}, a_{1}\right) \subset S_{\epsilon}\left(a_{1}\right)$ and hence $F_{p_{3}}\left(I_{1}\right)=I_{1}$. Consequently $L\left(p_{3}, I_{1}\right)=I_{2}$ is a convex 2-cell. Let $a_{2}$ denote the midpoint of $L\left(p_{3}, a_{1}\right)$, and note that $a_{2} \notin \partial I_{2}=I_{1} \cup L\left(p_{3}, \partial I_{1}\right)$.

Again, either $I_{2}$ contains a neighborhood of $a_{2}$, or it does not. If if does not, we proceed as above until we obtain a convex $n$-cell, $I_{n}$, and a point $a_{n} \epsilon$ $I_{n}-\partial I_{n}=I_{n}-$ bdry $I_{n}$ such that $I_{n}$ contains a neighborhood of $a_{n}$. And since $a_{n} \notin \partial I_{n}, a_{n} \notin \partial X$.

Now if $x \in \partial X$, by Theorem $4, L\left(a_{n}, x\right)$ is a maximal ray from $a_{n}$ in $X$. Hence if follows from Proposition 4 that $L\left(a_{n}, x\right) \cap I_{n}$ is a maximal ray from $a_{n}$ in $I_{n}$. Hence $L\left(a_{n}, x\right) \cap I_{n}=L\left(a_{n}, y\right)$ where $L\left(a_{n}, y\right) \cap \partial I_{n}=\{y\}$.

Conversely if $y_{0} \in \partial I_{n}$, then $L\left(a_{n}, y_{0}\right)$ is a maximal ray in $I_{n}$ with $L\left(a_{n}, y_{0}\right) \cap \partial I_{n}=\left\{y_{0}\right\}$ and if $L\left(a_{n}, x_{0}\right)$ is the maximal ray in $X$ containing $L\left(a_{n}, y_{0}\right)$, then $L\left(a_{n}, x_{0}\right) \cap \partial X=\left\{x_{0}\right\}$.

Now we define $f: X \rightarrow I_{n}$ as follows: $f\left(a_{n}\right)=a_{n}$; and if $x \neq a_{n}$, let $L\left(a_{n}, x_{0}\right)$ be the maximal ray in $X$ from $a_{n}$ through $x$ and $L\left(a_{n}, y_{0}\right)=I_{n}$ ก $L\left(a_{n}, x_{0}\right)$ where $x_{0} \in \partial X$ and $y_{0} \in \partial I_{n}$, then $f(x)$ is that point on $L\left(a_{n}, y_{0}\right)$ which is the image of $x$ under the mapping which takes $L\left(a_{n}, x_{0}\right)$ linearly on $L\left(a_{n}, y_{0}\right)$ with $f\left(a_{n}\right)=a_{n}$ and $f\left(x_{0}\right)=y_{0}$. 
Explicitly, if $\lambda\left(a_{n}, x_{0}, r\right)=x, 0 \leq r \leq 1$, then $f(x)=\lambda\left(a_{n}, y_{0}, r\right)$. Now $f$ is a $1-1$ function of $X$ onto $I_{n}$ taking $\partial(X)$ onto $\partial I_{n}$.

In view of the continuity of $\lambda$ and the fact that $\partial I_{n}=$ bdry $I_{n}$ is closed, the continuity of $f$ results from the following:

Lemma. If $\left\{p_{i}\right\}_{1}^{\infty} \rightarrow p_{0}$ and $p_{i} \neq a_{n}, i \geq 0$, and if $L\left(a_{n}, x_{i}\right)$ is the maximal ray in $X$ from $a_{n}$ through $p_{i}, i \geq 0$, then $\left\{x_{i}\right\}_{1}^{\infty} \rightarrow x_{0}$.

Proof. Let $\left\{x_{n_{i}}\right\}_{1}^{\infty} \rightarrow x_{0}^{\prime}$ be any convergent subsequence of $\left\{x_{i}\right\}_{1}^{\infty}$. We show $x_{0}^{\prime}=x_{0}$. Since $\left\{L\left(a_{n}, x_{n_{i}}\right)\right\} \rightarrow L\left(a_{n}, x_{0}^{\prime}\right), p_{0} \in L\left(a_{n_{n}}, x_{0}^{\prime}\right)$. Hence $L\left(a_{n}, x_{0}^{\prime}\right) \subset$ $L\left(a_{n_{i}}, x_{0}\right)$. Since $\left\{x_{i}\right\}_{0}^{\infty} \subset \partial X$ and $\partial X$ is closed, $x_{0}^{\prime} \in \partial X$. Hence, again by Theorem $4, x_{0}^{\prime}=x_{0}$.

III. Questions. It is an open question, asked by Borsuk and Bing, whether or not each finite dimensional compact metric space $X$ which admits an $\alpha$-metric is homeomorphic to a cell. If $\operatorname{dim} X=n$, then Lelek and Nitka [7] supply an affirmative answer if $n \leq 2$. Rolfsen [3] proves that $X$ is a cell if $n=3$; and he also shows that if $X$ is a manifold and $n>5$, then $X$ is a cell.

Question 1. If $X$ is a finite dimensional compact space with $\alpha$-metric $d$, can one use $d$ to define a $\beta$-metic on $X$ ?

Question 2. Is each compact infinite dimensional space $X$ with an $\alpha$-metric homeomorphic to the Hilbert cube?

Question 3. Same as above but assuming the existence of a $\beta$-metric on $X$.

Question 4. Is there a $\beta$-metric on the Hilbert cube such that each point is a rim point?

\section{REFERENCES}

1. R. H. Bing, Partitioning a set, Bull. Amer. Math. Soc. 55 (1949), 1101-1110. MR 11, 733.

2. J. L. Kelley, Hyperspaces of a continuum, Trans. Amer. Math. Soc. 52 (1942), 2236. MR 3, 315.

3. Dale Rolfsen, Strongly convex metrics in cells, Bull. Amer. Math. Soc. 74 (1968), 171-175. MR 37 \#2180.

4. - Geometric methods in topological spaces, Proc. Arizona State Univ. Topological Conf., Tempe, Ariz., 1967, pp. 250-257.

5. R. M. Schori, Hyperspaces and symmetric products of topological spaces, Fund. Math. 63 (1968), 77-87.

6. R. M. Schori and J. E. West, $2^{I}$ is homeomorphic to the Hilbert cube, Bull. Amer. Math. Soc. 78 (1972), 402-406.

7. A. Lelek and W. Nitka, On convex metric spaces. I, Fund. Math. $49(1960 / 61)$, 183-104. MR 23 \#A2192.

DEPARTMENT OF MATHEMATICS, UNIVERSITY OF MIAMI, CORAL GABLES, FLORIDA 33124 